PROCEEDINGS OF THE

AMERICAN MATHEMATICAL SOCIETY

Volume 125, Number 11, November 1997, Pages 3133-3135

S 0002-9939(97)04274-3

\title{
A REMARK ON THE LIFTABLE DERIVATION OF MODULI ALGEBRAS OF ISOLATED HYPERSURFACE SINGULARITIES
}

\author{
HAO CHEN
}

(Communicated by Eric M. Friedlander)

\begin{abstract}
An example is given to show that not every derivation in the nilradical of the Lie algebra of derivations of moduli algebras can be liftable and the dimension of the nilradical of the Lie algebra of derivations of moduli algebras is not a topological invariant for an isolated hypersurface singularity.
\end{abstract}

Let $(V, 0)=\left\{\left(z_{0}, z_{1}, \ldots, z_{n}\right) \in C^{n+1}, f\left(z_{0}, z_{1}, \ldots, z_{n}\right)=0\right\}$ be an isolated hypersurface singularity at the origin defined by a polynomial $f\left(z_{0}, z_{1}, \ldots, z_{n}\right)$, and let $A(V)=C\left\{z_{0}, z_{1}, \ldots, z_{n}\right\} /\left(f, \partial f / \partial z_{0}, \partial f / \partial z_{1}, \ldots, \partial f / \partial z_{n}\right)$ be its moduli algebra. It is proved in [1] by J. Mather and Stephen Yau that the moduli algebras can be used to classify the analytic structures of singularities. In [4] finite dimensional Lie algebras $L(V)$ of derivations on moduli algebras were introduced and these Lie algebras $L(V)$ were proved to be solvable in a series of later work of Stephen Yau ([5] and [6]). This first established the link between the theory of isolated hypersurface singularities and the theory of solvable Lie algebras; in particular, one can expect to construct invariants of isolated hypersurface singularities via those solvable Lie algebras $L(V)$. In [2] continuous analytic invariants for $\widetilde{E}_{7}$ and $\widetilde{E}_{8}$ singularities have been constructed from the Lie algebras $L(V)$ of derivations on moduli algebras and some Torelli type theorems have been proved. The advantage in the $\widetilde{E}_{7}$ and $\widetilde{E}_{8}$ cases is that the nilradicals of $L(V)$ are in the $\widetilde{L}(V)$ (for definition, see $[2])$, that is, every derivation in the nilradical of those $L(V)$ can be lifted. Actually the construction in [2] depends on the bracket operations in the nilradical of those $L(V)$. In this note we give an example in which case not every derivation in the nilradical of $L(V)$ is liftable. In [3] the generalized Cartan matrices, the stable derivation algebras and the nilradicals of $L(V)$ for simple elliptic singularities $\widetilde{E}_{6}$, $\widetilde{E}_{7}$ and $\widetilde{E}_{8}$ were computed. It is shown that the generalized Cartan matrices and the stable derivation algebras are not topological invariants, but the dimensions of nilradicals for those three class singularities are topological invariants. However in our example here it is also indicated that the dimension of the nilradical of $L(V)$ is not the topological invariant.

Notation (as in [2]):

$(V, 0)$ : an isolated hypersurface singularity at the origin;

Received by the editors May 11, 1994.

1991 Mathematics Subject Classification. Primary 14B05.

The author's research was supported by NNSF of China.

(C)1997 American Mathematical Society 
$A(V)$ : the moduli algebra of $(V, 0)$;

$L(V)$ : Lie algebras of derivations on $A(V)$;

$\left(V_{t}, 0\right)$ : a family of isolated hypersurface singularities;

$\widetilde{L}\left(V_{t_{0}}\right)$ : Lie subalgebra of $L\left(V_{t_{0}}\right)$ of liftable derivations; a derivation $D_{t_{0}}$ is liftable means that it is the special value at $t_{0}$ of a family of derivations $D_{t}$ in $L\left(V_{t}\right)$ (see $[2])$.

Let $\left(V_{t}, 0\right)=\left\{(x, y, z) \in C^{3}, f_{t}(x, y, z)=x^{4}+y^{4}+z^{4}+t x^{2} y^{2}\right\}$ be a family of isolated hypersurface singularities of dimension 2 , and let $t \in C-\{ \pm 2\}$ be the parameters. The family is topologically constant. It is easy to check that the following monomials are the $C$-linear base for the moduli algebras $A\left(V_{t}\right)$ :

$$
\begin{gathered}
1 \\
x, y, z \\
x^{2}, y^{2}, z^{2}, x y, y z, z x \\
x^{2} y, x^{2} z, y^{2} x, y^{2} z, z^{2} x, z^{2} y, x y z \\
x^{2} y^{2}, x^{2} z^{2}, y^{2} z^{2}, x^{2} y z, y^{2} x z, z^{2} x y \\
x^{2} y^{2} z, x^{2} z^{2} y, y^{2} z^{2} x \\
x^{2} y^{2} z^{2}
\end{gathered}
$$

The moduli algebra $A\left(V_{t}\right)$ has the above $C$-linear base with the following multiplication table:

$$
\begin{gathered}
x^{3}=(-1 / 2) t x y^{2}, \quad y^{3}=(-1 / 2) t x^{2} y, \quad z^{3}=0, \\
x^{4}=(-1 / 2) t x^{2} y^{2}, \quad y^{4}=(-1 / 2) t x^{2} y^{2}, \quad z^{4}=0, \\
z^{3} y=z^{3} x=x^{3} y=y^{3} x=0, \\
x^{3} z=(-1 / 2) t y^{2} x z, \quad y^{3} z=(-1 / 2) t x^{2} y z .
\end{gathered}
$$

Let

$$
\begin{aligned}
D_{t}= & \left(\sum_{i+j+k=2} a_{i j k}(t) x^{i} y^{j} z^{k}\right) \partial / \partial x+\left(\sum_{i+j+k=2} b_{i j k}(t) x^{i} y^{j} z^{k}\right) \partial / \partial y \\
& +\left(\sum_{i+j+k=2} c_{i j k}(t) x^{i} y^{j} z^{k}\right) \partial / \partial z
\end{aligned}
$$

be a derivation in $L\left(V_{t}\right)$ where $a_{i j k}(t), b_{i j k}(t)$ and $c_{i j k}(t)$ are analytic functions of the parameter $t$. The necessary and sufficient condition for $D_{t}$ in $L\left(V_{t}\right)$ is that $D_{t}$ keeps the Jacobian ideal $J_{t}=\left(\partial f_{t} / \partial x, \partial f_{t} / \partial y, \partial f_{t} / \partial z\right)$ invariant. The direct computation with the help of the multiplication table (2) indicates that the condition $D_{t}\left(J_{t}\right) \subset J_{t}$ is equivalent to the following conditions about $a_{i j k}(t), b_{i j k}(t), c_{i j k}(t)$ :

$$
\begin{gathered}
a_{002}(t)=b_{002}(t)=c_{002}(t)=c_{020}(t)=c_{200}(t)=0, \\
4 t a_{101}(t)-4 t b_{011}(t)=0 \\
\left(12-t^{2}\right) a_{011}(t)+4 t b_{101}(t)=0 \\
4 t a_{011}(t)+\left(12-t^{2}\right) b_{101}(t)=0
\end{gathered}
$$




$$
\begin{aligned}
\left(12-t^{2}\right) a_{020}(t)+4 t b_{110}(t)-4 t a_{200}(t) & =0, \\
\left(12-t^{2}\right) b_{200}(t)+4 t a_{110}(t)-4 t b_{020}(t) & =0 .
\end{aligned}
$$

Note that in equation (6), the conditions on $a_{011}(t)$ and $b_{101}(t)$ are not invariant when the parameter $t$ changes. For any parameter $t \in C-\{ \pm 2,6\}$ equation (6) implies $a_{011}(t)=b_{101}(t)=0$, and for $t=6$ equation (6) implies $a_{011}(6)=b_{101}(6)$. This means $D_{6}=y z \partial / \partial x+x z \partial / \partial y$ is in $L\left(V_{6}\right)$ but not liftable. Actually if $D_{6}$ is liftable it would have to be the special value at 6 of a family of derivations $D_{t}$ in $L\left(V_{t}\right)$. By the analyticity of coefficients we have that the $a_{011}(6)$ and $b_{101}(6)$ of this $D_{6}$ have to be zero. This is a contradiction. Thus this $D_{6}=y z \partial / \partial x+x z \partial / \partial y$ is not liftable.

On the other hand the above $D_{6}$ is of pure degree 1 . Thus it is in the nilradical of $L(V)$ and we give an example which shows that the derivation in the nilradical may not be liftable.

A similar computation for the degree 0 derivations shows that $L\left(V_{t}\right)_{0}$ is spanned by the Euler operator $E=x \partial / \partial x+y \partial / \partial y+z \partial / \partial z$ except when $t=0$. Hence the nilradicals of $L\left(V_{t}\right)$ are just those derivations of degree $\geq 1$. Our example also indicates that the dimension of the nilradical of $L\left(V_{6}\right)$ is strictly greater than the dimensions of nilradicals of those $L\left(V_{t}\right)$ for $t$ in a sufficiently small neighborhood of 6 when $t=6$ is deleted. This gives the example in which the dimension of the nilradical of $L\left(V_{t}\right)$ is not topologically invariant.

\section{ACKNOWLEDGEMENT}

The author is grateful to Professor Stephen S-T. Yau for fruitful discussions about this problem.

\section{REFERENCES}

1. J. Mather and Stephen S-T. Yau, Classification of isolated hypersurface singularities by their moduli algebras, Invent. Math. 69 (1982), 243-251. MR 84c:32007

2. C. Seeley and Stephen S-T. Yau, Variation of complex structure and variation of Lie algebras, Invent. Math. 99 (1990), 545-565. MR 90k:32067

3. C. Seeley and Stephen S-T. Yau, Algebraic method in the study of simple elliptic singularities, Proc. of U.S.-U.S.S.R. Algebraic Geometry Symposium, Lecture Notes in Math. 1479 (1991), Springer-Verlag, 216-237. MR 94c:14002

4. Stephen S-T. Yau, Solvable Lie algebras and generalized Cartan matrices arising from isolated singularities, Math. Zeit. 191 (1986), 489-506. MR 87k:32014

5. Stephen S-T. Yau, Singularities defined by sl $(2, C)$ invariant polynomials and solvability of Lie algebras arising from isolated singularities, Amer. J. Math. 108 (1986), 1215-1240. MR 88d:32022

6. Stephen S-T. Yau, Solvability of Lie algebras arising from isolated singularities and nonisolatedness of singularities defined by $\operatorname{sl}(2, C)$ invariant polynomials, Amer. J. Math. 113 (1991), 773-778. MR 92j:32125

Department of Mathematics, Zhongshan University, Guangzhou, Guangdong 510275, People's Republic of China 